\title{
Impact of Laboratory Diagnostic Preanalytics on Patient Blood Management
}

\author{
Kathrin Schlatterer ${ }^{1,2, *}$, Kathrin Schlüter ${ }^{3}$ \\ ${ }^{1}$ Department of Laboratory Medicine, Sankt Gertrauden-Krankenhaus, Berlin, Germany \\ ${ }^{2}$ Institute of Clinical Chemistry and Laboratory Medicine, University Medicine of Greifswald, Greifswald, Germany \\ ${ }^{3}$ BD Life Sciences, Becton Dickinson GmbH, Heidelberg, Germany
}

Email address:

kathrin.schlatterer-krauter@sankt-gertrauden.de(K. Schlatterer),kathrin.schlueter@bd.com (K. Schlüter)

${ }^{*}$ Corresponding author

\section{To cite this article:}

Kathrin Schlatterer, Kathrin Schlüter. Impact of Laboratory Diagnostic Preanalytics on Patient Blood Management. International Journal of Clinical and Experimental Medical Sciences. Vol. 5, No. 2, 2019, pp. 33-40. doi: 10.11648/j.ijcems.20190502.12

Received: March 14, 2019; Accepted: May 15, 2019; Published: June 29, 2019

\begin{abstract}
This study was designed to investigate the possibility of reducing diagnostic blood loss as a lab-side element of Patient Blood Management. To this end, the nominal blood volume of blood collection tubes was reduced and, additionally, preanalytical processes were optimised to minimise repeated blood collections. These interventions were conducted in conjunction with a change of blood collection system. Auditing the preanalytical phase and observing sample quality before and after the interventions enabled the assessment of changes in preanalytical quality. The occurrence of several blood collection non-compliances had decreased substantially one year after preanalytical training and conversion to the different blood collection system. Improvement of preanalytical quality combined with reduction of tube blood volumes led to a significant decrease in the volume of blood drawn for diagnostic purposes, corresponding to a reduction of $177 \mathrm{~L}$ per year for the whole hospital. We also observed a reduction in the total number of blood collection tubes used. An analysis of data for geriatric and Intensive Care Unit (ICU) ward types, where there is a known risk of iatrogenic anaemia, revealed significant blood savings. In conclusion, targeted preanalytical training measures and introduction of blood collection tubes with lower draw volumes enable significant reductions in the volume of patient blood drawn for laboratory diagnostics, and so represent an important contributor to patient blood management.
\end{abstract}

Keywords: Preanalytical Phase Audit, Sample Quality, Diagnostic Blood Loss, Patient Blood Management

\section{Introduction}

In all industrial countries, the willingness to donate blood has, for a variety of reasons, decreased gradually over a number of decades. This has led to a reduction in the availability of blood products for allogeneic blood transfusions. Together with an increasing awareness of blood transfusion-related immunomodulation (TRIM), this has led to the necessity of a reduction in the use of blood transfusions that has been achieved via different structured approaches. The Patient Blood Management concept (PBM) is one of these and has been promoted by the WHO since 2011 [1].

In this context, the main focus has been on presurgical examination for the detection and adequate treatment of anaemia as well as efficient surgical coagulation management, with the goal of reducing intra- and postsurgical erythrocyte transfusions. Blood collected for laboratory diagnostics is known to have a significant impact on total blood loss [2]. Depending on the severity and duration of diseases, diagnostic blood loss can induce iatrogenic anemia and increase the need for blood transfusions [3]. Therefore, an institution's preanalytical phase processes and especially its blood collection strategy should be critically reviewed at regular intervals. Due to analytical methodological developments, blood volumes needed for blood tests have steadily decreased over a number of decades [4]. Moreover, avoidance or reduction of preanalytical errors can improve sample quality and therefore prevent unneccessary (repeated) blood collections and improve laboratory analysis turnaround-time, which may 
reduce the need for the additional use of point of care patient testing (POCT) [5].

The overall aim for blood collections should be to ensure consistently high analytical quality while minimizing the blood volume needed for diagnostic purposes.

Therefore, as part of a planned conversion of the blood collection system in our hospital, the complete preanalytical process was reviewed in order to identify areas for improvement. A preanalytical phase audit revealed noncompliances during hospital blood collections and also sample quality issues. A plan was developed to improve preanalytical quality, establish a contemporary low-volume blood collection strategy and thereby increase hospital efficiency. This enabled a multimodal, targeted approach (Figure 1).

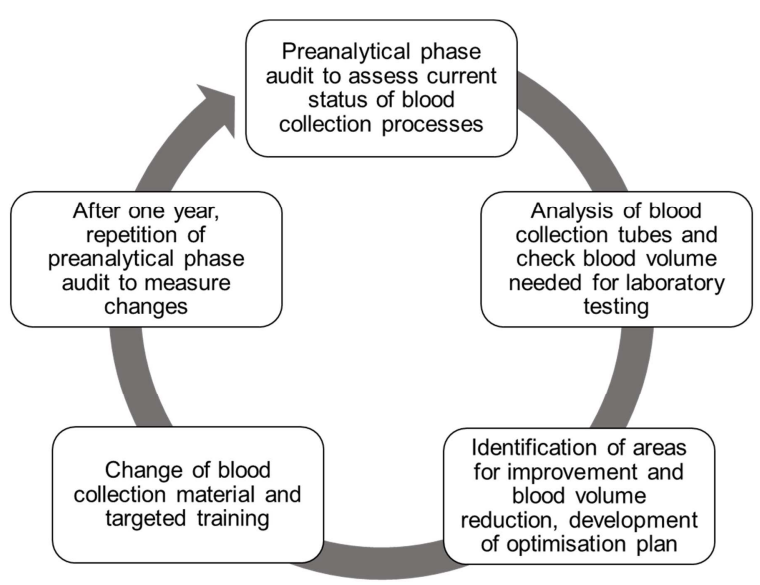

Figure 1. PDCA-Cycle in project plan.

The reduction in the volume of blood collected as a result of improved preanalytics was determined from the number of blood tubes used within the hospital over one year and from patient numbers. The volumes of blood saved were quantified for geriatric patients and for Intensive Care Unit (ICU) patients over a period of 4 weeks.

\section{Material and Methods}

The aim of the initial preanalytical phase audit was to determine the degree of compliance of preanalytical processes with widely accepted best practices and thus identification of areas for improvement. Using BD Laboratory Consulting Services $^{\circledR}$, preanalytical errors were investigated that have the potential to reduce blood sample quality and thereby lead to unnecessary repeated blood collections and a consequent delay in diagnosis. Tourniquet time was documented to be inappropriate if the tourniquet was not released before the second tube was drawn. Minimum requirement for tube mixing was 3 complete inversions. In addition, hygiene control measures and observed non-compliances leading to an increased risk of needle stick injuries during and after blood collection were noted. After disinfection, the puncture site should not be touched again and the disinfectant should be dry before puncturing the vein. The blood collection needle safety mechanism should be activated according to the manufacturer's instructions for use immediately after blood collection. Sharps should be disposed off without delay and without the need to walk to the sharps container. For patient identification, a minimum requirement of 3 different nonleading questions was set. In 6 representative hospital departments, including the ER, 54 blood collections were observed by trained specialists, using standardized questionnaires. In the central laboratory, 563 blood samples were visually inspected for hemolysis, latent clotting, clot formation and fill volume. Hemolysis was judged visually using the colour scale published by Dugan et al [6]. Tubes where the addititve on the label did not correspond with the additive on the tube or incorrect label positioning on the tube were documented as these lead to additional work load and increases in turnaround time (TAT) in the laboratory. A plan for process optimization was established based on the preanalytical errors detected.

Preparation for the change of blood collection system was conducted at departmental level across the whole clinic by personal interviews, addressing physicians concerns' resulting from a change in practice. Two kick-off events for a bigger audience consisting of a wide range of medical staff were held in order to raise awareness of the preanalytical errors observed during the audit and the implications of these errors on laboratory diagnostics, creating a sense of urgency (Kotter Change Management Model [7]). In addition, the conversion schedule was explained. The aim of these events was to gain acceptance for the changeover process.

The blood collection system was converted from a Monovette $^{\circledR}$-system to BD Vacutainer ${ }^{\circledR}$, introducing gel-tubes and tubes with a lower nominal fill volume. When changing the blood collection system, the main focus of the training was to address the preanalytical errors identified in the initial audit. After the conversion, there was a monitoring phase of approximately three months that was used to identify departments with additional training needs and to meet those needs. One year after conversion, a re-audit was performed, reviewing the same aspects as in the initial audit for 48 blood collections, and inspecting 534 blood samples visually in the central laboratory.

As an indirect measure for the effectiveness of preanalytical training and avoidance of preanalytical errors, leading to less repeated blood collections, the number of tubes used hospital-wide for each blood collection system per year was determined and correlated with patient numbers per year. The total volume of patients' blood collected was calculated for the Monovette ${ }^{\circledR}$ and the BD Vacutainer ${ }^{\circledR}$ systems.

In order to quantify blood savings in patient groups especially prone to development of iatrogenic anemia, two departments, geriatrics and intensive care, were analysed in more depth. A statistical analysis was performed, using the laboratory information system and checking all laboratory assignments in these departments over a 1 month time period before and for a 1 month period 1 year after the change in blood collection system. The timing of the latter was to 
ensure that on the one hand, preanalytical errors leading to repeated blood collections due to a familiarization period with the new system were excluded and, on the other hand, to exclude a possible opposite effect of extra diligence when handling a new, unfamiliar system resulting in fewer repeated blood collections. For geriatric patients, the numbers of blood tubes per patient per day and week were determined and the average blood collection volume per patient per week was compared before and after the conversion (86 and 84 patients, respectively). For the intensive care patients, a stay in ICU of at least 7 days was set as the filter criterion. For the 1 month query period prior to the introduction of the different collection system, there were 32 patients that met this criterion for the Monovette ${ }^{\circledR}$ system. For the 1 month query period 1 year after the introduction of the BD Vacutainer ${ }^{\circledR}$ system, there were 33 patients that met this criterion. All individual laboratory assignments for these patients were checked during each patient's complete ICU stay and the corresponding blood tubes, which entry in the laboratory was confirmed by means of a laboratory entry scan, were determined. Blood volume drawn for diagnostic purposes during each patient's complete ICU stay was calculated on this basis and compared between the two blood collection systems. The average blood volume collected was calculated per patient and week.

\section{Results}

\subsection{Preanalytical Efficiency of Blood Sampling in Different Departments}

\subsubsection{Blood Collection Material Storage}

There were no significant non-compliances with respect to blood collection material storage conditions in the initial preanalytical audit. However, four blood collection tubes were found that were beyond their expiry date and resulted in the need to repeat the blood collection. As a result of this finding, a "first-in-first-out"-methodology was implemented in the standard operation procedures for medical staff. One year after conversion, no expired tubes were found on the wards inspected.

\subsubsection{Patient Identification}

The omission of adequate patient identification carries a high risk for patient misidentification in daily work with consequences such as inadequate diagnosis or inappropriate treatment of patients. Therefore, it was noted whether three independent patient identification criteria were correctly checked with non-leading questions in the identification process. Training led to a significant improvement compared with the initial situation (3.7 fold) (Figure 2).

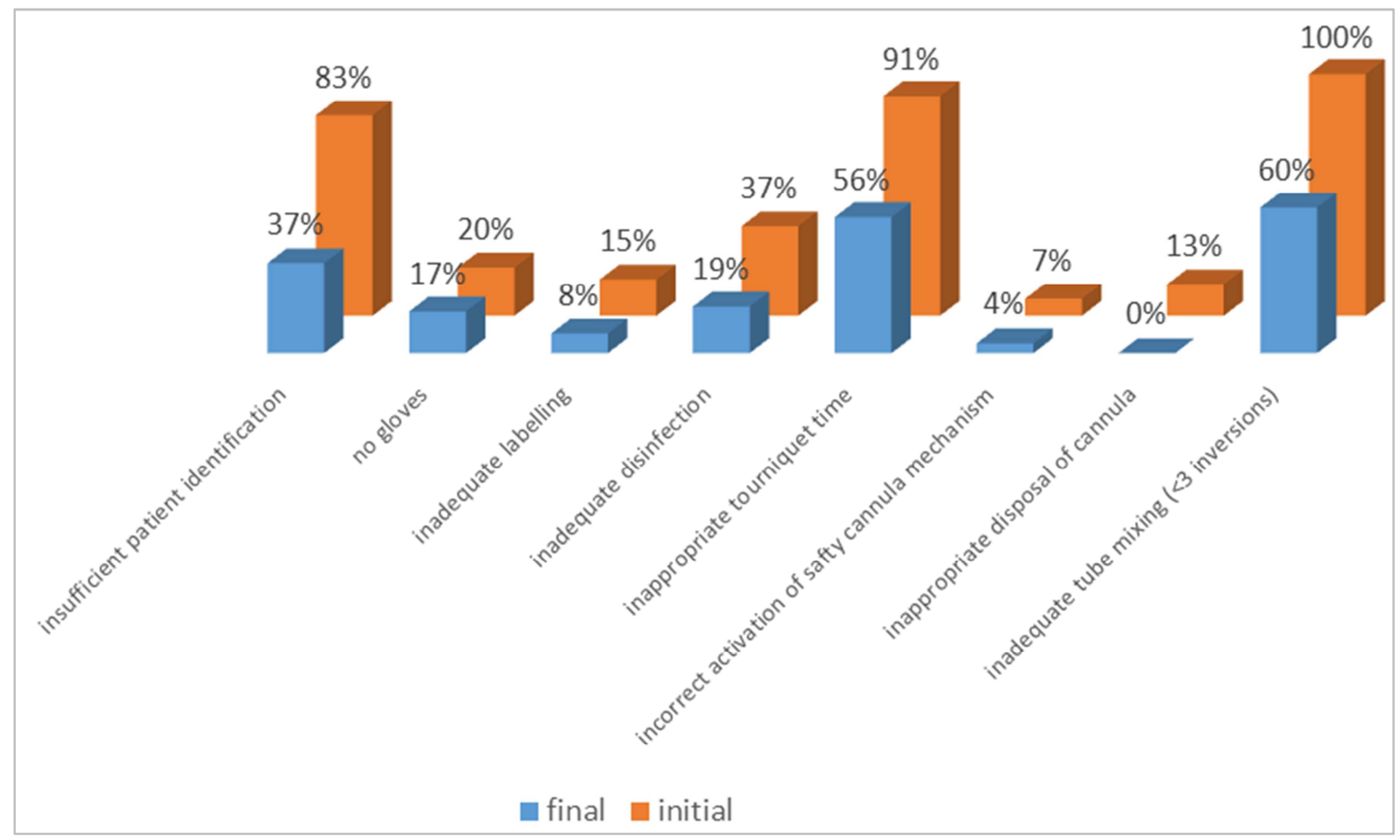

Figure 2. Improvement of preanalytical processes after training.

\subsubsection{Sample Identification}

Selection of the correct blood collection tube for the selected analysis is essential as is the correct labelling of the tubes with patient data, typically by applying barcode labels. The following non-compliances regarding blood tube labelling were recorded, which lead to a delay in laboratory analysis: label was glued at an oblique angle or in a position which did not follow manufacturer's recommendations, label stood out, label indicated a different additive to the actual tube type. All of these events lead to manual re-processing in the laboratory and thereby to a delay in diagnostics and unneccessary time ultilization of laboratory staff. Furthermore, re-labelling bears a significant risk of samples 
being associated with the wrong patient, with consequences for patient safety. After successful training and clarification of the importance of correct labelling for laboratory analysis turnaround time, the initial rate was almost halved (Figure 2).

\subsubsection{Collection Process, Hygiene and Avoidance of Needle Stick Injuries}

The percentage of blood collections where gloves were worn was almost the same before and after training. Incorrect disinfection of the puncture site was almost halved by training measures (Figure 2). The percentage of collections having an inappropriate tourniquet time decreased by $38 \%$ after training when compared with the initial audit. However, this number still offers potential for optimization (Figure 2) [8-9]. Initially, blood tubes were not mixed sufficiently (at least 3 inversions) in any of the blood collections. This noncompliance was decreased by $40 \%$ following the training, which also leaves further opportunity for optimization (Figure 2). In both the initial audit and the re-audit, cannulae with a safety mechanism were used. There was a very low rate of incorrect activation of the safety mechanism before and a slightly lower rate after conversion, but the difference was not significant (Figure 2). Incorrect, delayed disposal of the cannulae, sometimes even with forcing the needle into an overfilled disposal container by hand, was no longer observed after the training (Figure 2).

\subsection{Preanalytical Efficiency Observed in the Central Laboratory}

Blood collection tubes of different sample types from the initial audit $(n=563)$ and from the re-audit $(n=534)$ were inspected for sample quality and observed from their arrival in the laboratory until they were loaded into the appropriate analyzer. Initially, serum and lithium heparin tubes without separation gel were used. For reasons of improved stability of analytes and a higher sample yield, all non-gel tubes for clinical chemistry analyses were replaced by gel tubes during the conversion process. In order to minimize the blood volume drawn, the nominal volumes of the blood collection tubes were adjusted (Figure 3 ).

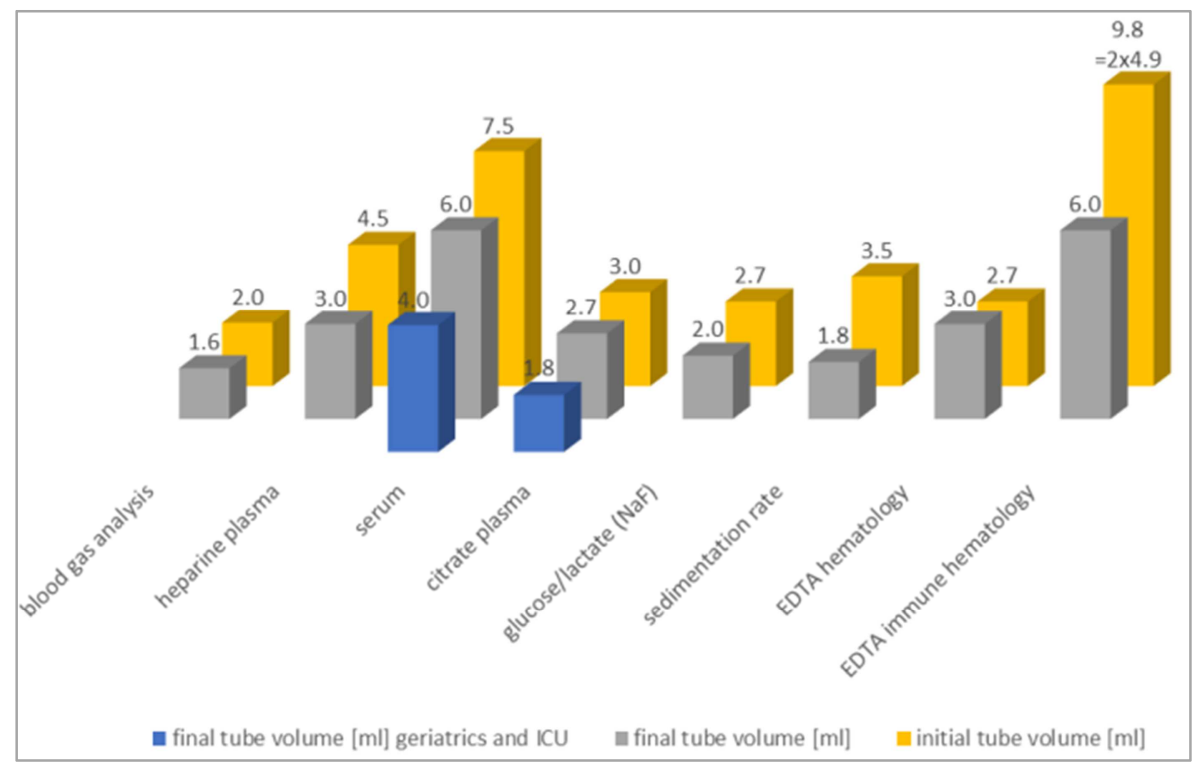

Figure 3. Change in tube volumes.

\subsubsection{Correct Fill Volume of Tubes}

As shown in Figure 2, the initial rate of citrate tubes filled below 90\% was already low. This had been achieved by continuous training and feedback using the hospital's quality management system. Underfilling rates of citrate tubes 1 year after the conversion had slightly increased. The rate of ethylenediaminetetraacetic acid (EDTA) tubes filled below $75 \%$ also increased slightly. The rate of clinical chemistry analysis serum tubes filled below $75 \%$ decreased slightly. In the case of lithium heparin tubes, the change was very impressive. The rate of tubes filled below $75 \%$ decreased by $70 \%$ (Figure 4). Further continuous training is a mandatory part of the quality management system.

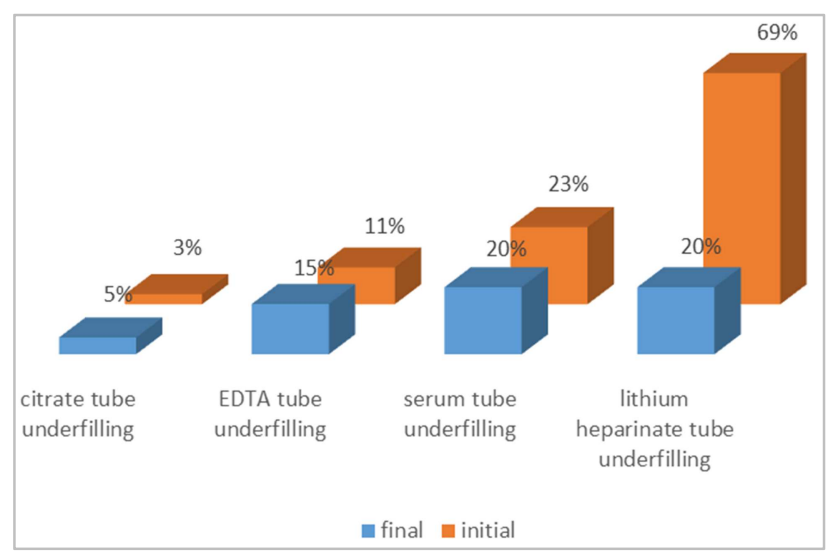

Figure 4. Change in underfilling rates after training. 


\subsubsection{Fibrin and Clotting Towers}

After conversion, the actual volume of serum available for testing approached the theoretically available serum volume, as there were no so-called clotting towers formed with the new serum gel tubes (Figure 5).

These towers, a specific form of clot spanning almost the length of the tube even after centrifugation of plain serum tubes, occurred regularly with the initial blood collection system, probably due to a combination of tube morphology and clotting of the sample in a horizontal tube position (Figure 5). To optimize sample yield, filters were inserted into the tubes to push the clot down and compress it manually. This involved extra time and sometimes made an additional centrifugation step necessary, when cells were resuspended during filter placement. Clotting towers were completely eliminated after conversion without changing the tube positioning during clotting (Figure 6).

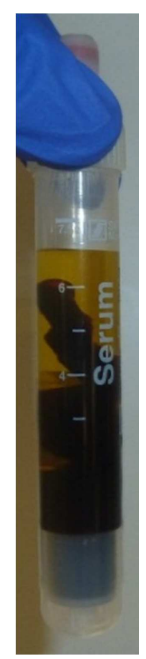

Figure 5. Clotting tower in serum tube.

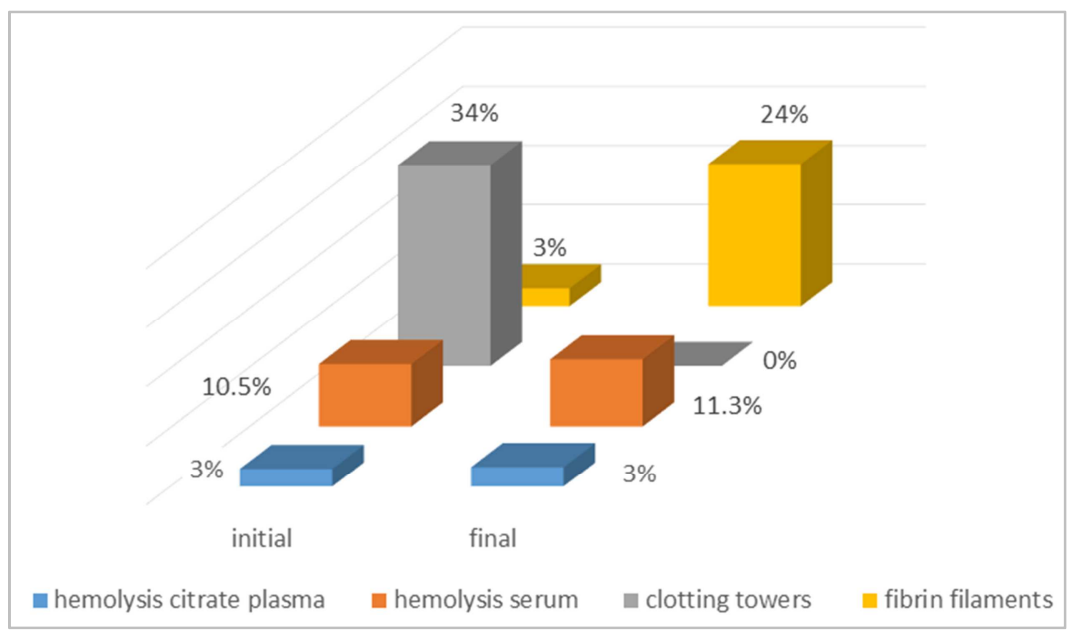

Figure 6. Change in hemolysis rates and regarding clotting due to insufficient tube mixing after training.

The detection of fibrin clouds and fibrin filaments on the other hand increased after conversion. Predominantly fibrin filaments were observed, only 2 cases showed fibrin clouds. This demonstrates that further training in tube processing, especially the importance of mixing the blood in serum tubes with the clotting activator, is required.

\subsubsection{Hemolysis}

Compared with the initial audit, no statistically significant change in hemolysis rates was found in the re-audit (Figure 6). This suggests that hemolysis rates in the two tube types are effectively the same.

\subsection{Quantification of Diagnostic Blood Loss}

Since the main focus of this study was the reduction in the volume of blood collected by introducing preanalytical improvements as well as by reduction of tube sizes, a detailed analysis of the impact of the changes was performed for the geriatric department and ICU, both caring for patients that are especially prone to the development of iatrogenic anaemia. These results combine training effects as well as tube volume change effects.

\subsubsection{Geriatric and ICU Patients}

Geriatric patients are rather vulnerable to iatrogenic anaemia due to their multimorbidity. For these patients, the reductions in blood volume drawn for laboratory diagnostics by reducing the size of the tubes were determined over a time period of 1 week before and after the change of blood collection system. The volume of blood drawn from geriatric patients during 1 week for diagnostic reasons was nearly halved (Figure 7). A further patient group that is prone to the development of iatrogenic anaemia are intensive care patients $[2,10,11]$. Patients with a longer stay in the ICU in particular have to undergo blood tests several times a day for monitoring. Blood sampling for point-of-care diagnostics, such as blood gas analysis, must be also be considered when calculating and attempting to reduce total diagnostic blood loss. Mean diagnostic blood loss for ICU patients after conversion was reduced by $27.2 \%$ per week (Figure 7 ). The fact that savings in the ICU were smaller than in geriatric patients is due to the high usage of daily POCT-blood gas analysis, which is specific for ICU patients. 


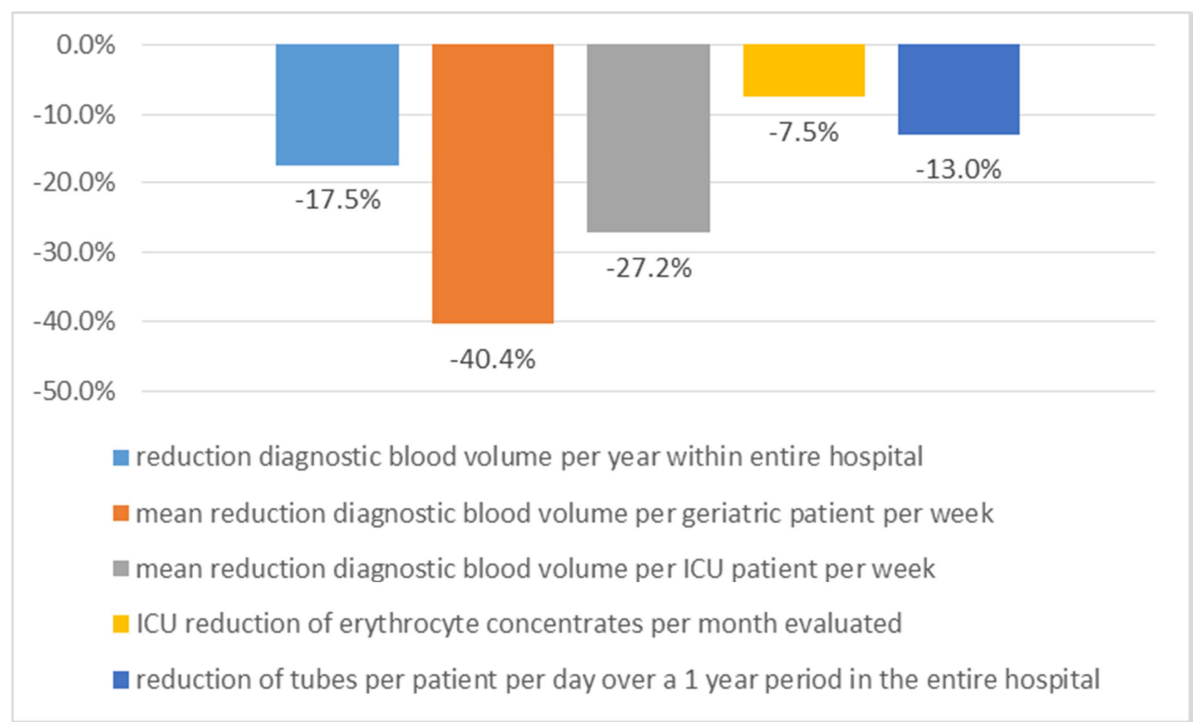

Figure 7. Reduction of diagnostic blood loss for entire hospital as well as for geriatric and ICU patients, Reduction of transfused erythrocyte concentrates in the ICU in evaluated month, reduction of tubes per patient and day in the entire hospital.

\subsubsection{Saving of Blood in the Entire Hospital}

For the complete hospital, a further quantification with respect to diagnostic blood collection was performed by comparing the total blood volume collected over 1 year using the Monovette ${ }^{\circledR}$ tubes with the volume collected over 1 year using the BD Vacutainer ${ }^{\circledR}$ tubes. This approach is an approximation as not all of the tubes that are taken from central storage and brought to the wards enter the diagnostic process. The number of tubes discarded for organizational reasons, such as use of blood collection tubes in training courses, blood collection failures, etc. cannot be excluded in this model. However, this applies equally to both blood collection systems and there were no changes that might be expected to cause a significant difference between the two assessment periods. It should be noted that the diagnostic blood volume using BD Vacutainer ${ }^{\circledR}$ system was evaluated 1 year after conversion. As medical staff become more familiar with the system and continuing training measures are undertaken, there could be further reductions in the number of blood collection tubes used. Calculations after additional periods will therefore be performed. A correlation with patient numbers per year revealed a significant saving in the mean number of blood collection tubes used per patient (Figure 7). The total increase of $1 \%$ in tubes per year after conversion was paralleled by a $4.9 \%$ increase in patient numbers and thereby constituted a reduction in the number of tubes per patient per day of $13 \%$ (Figure 7). In addition, nominal tube volumes were reduced (Figure 3). The total blood volume saved was averaged at approximately $177 \mathrm{~L}$ per year as calculated by the number of tubes used and the nominal filling volumes of the different tube types. This corresponds to a decrease in blood drawn for diagnostic purposes of $17.5 \%$ for all patients in the hospital (Figure 7).

\subsubsection{Extrapolation to the Theoretical Saving of Erythrocyte Concentrates for the Hospital}

Within the 1-month-period evaluated, the number of erythrocyte concentrates transfused in the ICU was decreased by $7.5 \%$ (Figure 7 ). Numbers of blood transfusions in the geriatric department are usually low, so the detection of significant differences was not possible. Due to the design of the study and the heterogeneity of patient groups in general, a correlation of reduced diagnostic blood loss with savings in transfused erythrocyte concentrates within the entire hospital is desirable but not readily possible.

However, a simple approximation is the conversion of the reduction of the overall blood volume collected into a theoretical saving of erythrocyte concentrates. With a volume of approximately $280 \mathrm{~mL}$ per transfused erythrocyte concentrate, the average saving of $177 \mathrm{~L}$ blood corresponds to a theoretical saving of 631 erythrocyte concentrates for the hospital per year $(-29.3 \%)$.

\section{Conclusion}

In the present study, a multimodal concept was established for minimizing diagnostic blood loss by decreasing the nominal draw volume of blood collection tubes and optimizing the preanalytical phase. In addition, this should have a positive impact on laboratory performance, both in terms of quality of results and turnaround time.

With this targeted approach, the initial situation was reviewed in order to identify the potential for optimization and then a multistep improvement strategy was implemented to increase efficiency and thereby improve patient care. Specific training measures accompanied the process, because preanalytics is a major pillar of high quality laboratory diagnostics but is only partially controlled by the diagnostic laboratory itself. Regular training and close supervision of blood collecting medical staff is required. Awareness of the importance of storage conditions, of the impact of correct disinfection measures, correct tourniquet time, avoidance of sample hemolysis, inadequate filling of tubes and correct mixing of tubes, were raised within a wider audience of 
medical staff using this approach. Demonstrating the actual level of non-compliances may have contributed to increasing the willingness to change habits.

The main possible causes of hemolysis are venipuncture through wet disinfectant, prolonged tourniquet time and drawing blood using a venous catheter [12-13]. Inadequate filling is one of the main reasons for repeated blood collections Underfilling, especially to the extent seen in heparin tubes from the Emergency Room as observed during the first audit, may be due to emergency situations and time pressure during blood collections. Additionally, even if the sample volume is sufficient for the test requested, sample quality may be compromised because of an increased concentration of the additive due to underfilling. This is best documented for citrate tubes, but there is also some evidence that high heparin concentrations can impact certain immunoassays [14].

There were two main causes of fibrin formation. One was insufficient mixing of serum tubes. Mixing is not only essential for lithium heparin, citrate and EDTA tubes, but also in serum tubes as it enables adequate contact of the blood with the clotting activator located on the tube wall and therefore prevents delayed coagulation, potentially leading to fibrin filaments. Secondly, clotting time may have been too short. Even small fibrin filaments can lead to technical problems by clogging instrument probes and thus to a delay in analysis.

Overall, using targeted training and constant communication, we achieved significant improvements in the preanalytical phase. This may be one reason contributing to the decrease in the number of tubes collected per patient per day. Awareness of the consequences of diagnostic blood loss may also have contributed. The other part of this study involved reducing blood collection volumes. In the case of blood collection systems currently in use in a hospital, small adjustments, such as the replacement of one type of tube by another, is possible in principle, but can be difficult to implement due to fixed procedures in purchasing processes. Thus, a general tube conversion of all tube types offers a good opportunity for making a significant change. The effect of blood collection volume reduction performed in this study was evaluated for geriatric as well as ICU patients, as both groups are vulnerable to developing iatrogenic anaemia caused by blood loss due to collections for laboratory diagnostics. A rough estimation of success was additionally performed for the entire hospital. Further independent studies using different patient groups could be conducted, as this study was conceived as a first attempt in quantifying blood savings achievable by such measures. Due to the relatively small groups included in the study and rising patient numbers within the hospital per year, a correlation with a potential general saving in transfusion of erythrocyte concentrates was not possible within the scope of this study. Preliminary data for ICU patients show that a correlation in principal seems possible. For this purpose larger study groups would be needed, especially as the medical backgrounds within ICU patients and geriatric patients is highly heterogeneous, thereby resulting in varying transfusion probabilities.

In particular, the analysis of blood saving possibilities in
ICU patients shows that understanding the benefits and drawbacks of blood gas analysis, easily performed as POCTanalysis and giving information on a variety of laboratory parameters besides blood gases, plays a key role in the task of decreasing diagnostic blood loss [15]. Due to its accessibility, point-of-care diagnostics are performed in parallel with central laboratory diagnostics, limiting the potential blood savings under the influence of a central laboratory. This demonstrates once again that, though it has undeniable advantages, the interactions of POCT with central laboratory diagnostics and the overall analysis process need to be carefully considered.

For this reason, TAT is important to minimize the noncritical use of POCT diagnostics. TAT is one of the most important and measurable characteristics for laboratories and for hospital benchmark purposes. It is also viewed by physicians and nurses as one of the most important measures for the service quality of the laboratory [16-17]. In particular, faulty preanalytical processes make a large contribution to a prolonged TAT. Therefore, an approach such as the one presented in this study can be used to influence the quality of such processes, which are normally outside the control of the laboratory.

The process optimization described in this study is an important building block in Patient Blood Management (PBM). In addition to a favourable individual effect on each patient, it could also reduce the need for transfusions and thus increase blood bank stocks to ensure that those patients who really need blood transfusions can receive them in the future. Improving preanalytical processes and reducing nominal tube collection volumes serve as a lab-side module of PBM for the prevention of hospital-induced anaemia by responsible handling of the patient's blood resource. The aim of multimodal PBM is to save the patient's blood resource. This is achieved by the recognition and adequate treatment of anaemia prior to surgical interventions, in order to reduce the likelihood of requiring a blood transfusion. In addition, it is also important to minimize the occurrence of anaemic conditions as a consequence of medical treatment. Blood losses by diagnostics, intervention and surgeries form a large proportion of hospital acquired anaemia conditions [18], so blood volumes required for laboratory diagnostics need to be considered. The more severely ill a patient is, the more intense laboratory diagnostics becomes and so more blood needs to be drawn for diagnostic purposes. Also, the efficiency of erythropoiesis is limited in seriously ill patients. As a result of these two factors, the risk of development of iatrogenic anaemia in this patient group is disproportionately increased.

This can be avoided by reducing diagnostic blood collection, by minimizing perisurgical blood loss, and by using evidence-based coagulation and hemotherapy concepts with a guideline-appropriate indication for administration of blood products. The impact of structured PBM in German hospitals has been the subject of multicentric studies. It was demonstrated that in addition to unambiguous short- and medium-term benefits for patients, there was also a total cost saving for hospitals [19-24]. In this context Fischer et al. 
indicated the importance of decreased diagnostic blood loss in 2014 [25-26].

The multimodal approach initiated by the laboratory as described here can be interpreted as a valuable lab-side contribution to PBM, whose effectiveness has been demonstrated by this study. Regarding PBM as a continuous optimization process, a possible next step for further volume reduction could be the implementation of computer-based laboratory control using strictly indication-related laboratory profiles.

\section{References}

[1] World Health Organization (2011) Global Forum for Blood Safety: Patient Blood Management. http://www.who.int/bloodsafety/events/gfbs_01_pbm_concept paper.pdf

[2] Wisser, D. van Ackern, K. Knoll, E. Wisser, H. and Bertsch T. (2003) Blood loss from laboratory tests. Clinical Chemistry, $49,1651-1655$.

[3] Ranasinghe, T. and Freeman, W. D. (2014) "ICU vampirism" - time for judicious blood draws in critically ill patients. British Journal of Haematology, 164, 302-303.

[4] Levi, M. (2014) Twenty-five million litres of blood into the sewer. Journal of Thrombosis and Haemostasis, 12, 1592.

[5] Spethmann, J. Schlüter, K. and Schlatterer, K. (2018) Laboratory medicine contributions to patient blood management concepts. Journal of Laboratory Medicine, 42, $81-87$.

[6] Dugan, L. Leech, L. Speroni, K. G. and Corriher, J. (2005) Factors affecting hemolysis rates in blood samples drawn from newly placed IV sites in the emergency department. Journal of Emergency Nursing, 31, 338-345.

[7] Kotter, J. P. (1996) Leading Change. Boston: Harvard Business School Press.

[8] Clinical and Laboratory Standards Institute (2007) Procedures for the collection of diagnostic blood specimens by venipuncture. PA: CLSI 6th ed. Wayne, H3-A6.

[9] World Health Organization (2010). WHO guidelines on drawing blood: best practices in phlebotomy. WHO Document Production Services, Geneva.

[10] Chant, C. Wilson, G. and Friedrich, J. O. (2006) Anemia, transfusion, and phlebotomy practices in critically ill patients with prolonged ICU length of stay: a cohort study. Critical Care, 10, R140.

[11] Tosiri, P. Kanitsap, N. and Kanitsap, A. (2010) Approximate iatrogenic blood loss in medical intensiv care patients and the causes of anemia. Journal of the Medical Association of Thailand, 93, 271-276.

[12] Lippi, G. Blanckaert, N. Bonini, P. Green, S. Kitchen, S. Palicka, V. et al. (2008) Haemolysis: an overview of the leading cause of unsuitable specimens in clinical laboratories. Clinical Chemistry and Laboratory Medicine, 46, 764-772.

[13] Lippi, G. Plebani, M. Di Somma, S. and Cervellin, G. (2011) Hemolyzed specimens: a major challenge for emergency departments and clinical laboratories. Critical Reviews in Clinical Laboratory Sciences, 48, 143-153.

[14] Tate, J. and Ward, G. (2004) Interferences in Immunoassay. The Clinical Biochemist Reviews, 25, 105-120.

[15] Hintereder, G. (2017) Patient blood management Labormedizinische Unterstützung und POCT. Journal of Laboratory Medicine, 41, 219-227.

[16] McCall, S. J. Souers, R. J. Blond, B. and Massie, L. (2016) Physician Satisfaction With Clinical Laboratory Services: A College of American Pathologists Q-Probes Study of 81 Institutions. Archives of Pathology and Laboratory Medicine, 140, 1098-1103.

[17] Jones, B. A. Walsh, M. K. and Ruby, S. G. (2006) Hospital nursing satisfaction with clinical laboratory services: a College of American Pathologists Q-Probes study of 162 institutions. Archives of Pathology and Laboratory Medicine, 130, 1756-1761.

[18] Quaseem, A. Alguire, P. Dallas, P. Feinberg, L. E. Fitzgerald, F. T. Horwitch, C. et al. (2012) Appropriate use of screening and diagnostic tests for foster high-value, cost-conscious care. Annals of Internal Medicine, 156, 47-49.

[19] Farmer, S. L. Towler, S. C. Leahy, M. F. and Hofmann, A. (2013) Drivers for change: Western Australia Patient Blood Management Program (WA PBMP), World Health Assembly (WHA) and Advisory Committee on Blood Safety and Availability (ACBSA). Best Practice \& Research: Clinical Anaesthesiology, 27, 43-58.

[20] Meybohm, P. Fischer, D. P. Geisen, C. Müller, M. M. Weber, C. F. Herrmann, E. et al. (2014) Safety and effectiveness of a Patient Blood Management (PBM) program in surgical patients--the study design for a multi-centre prospective epidemiologic non-inferiority trial. BMC Health Services Research, 14, 576.

[21] Meybohm P. Herrmann E. Steinbicker A. U. Wittmann M. Gruenewald M. Fischer D. et al. (2016) Patient Blood Management is Associated With a Substantial Reduction of Red Blood Cell Utilization and Safe for Patient's Outcome: A Prospective, Multicenter Cohort Study With a Noninferiority Design. Annals of Surgery, 264, 203-211.

[22] Zacharowski K. and Spahn D. R. (2016) Patient Blood Management equals patient safety. Best Practice \& Research: Clinical Anaesthesiology, 30, 159-169.

[23] Fischer D. P. Zacharowski K. Müller M. M. Geisen C. Seifried E. Müller H. et al. (2015) Patient Blood Management implementation strategies and their effect on physicians' risk perception, clinical knowledge and perioperative practice - the Frankfurt experience. Transfusion Medicine and Hemotherapy, 42, 91-97.

[24] Müller M. M. Fischer D. Stock U. Geisen C. Steffen B. Nussbaumer J. et al. (2014) Patient Blood Management-The preoperative patient. Anästhesiologie, Intensivmedizin, Notfallmedizin und Schmerztherapie, 49, 246-253.

[25] Fischer D. Geisen C. Steffen B, Meybohm P. Schmitz-Rixen T. (2014) Patient Blood Management-The inpatient care. Anästhesiologie, Intensivmedizin, Notfallmedizin und Schmerztherapie, 49, 256-264.

[26] Fischer D. P. Zacharowski K. Meybohm P. (2014) Savoring every drop - vampire or mosquito? Critical Care, 18, 306. 